\title{
Nursing in patients with hematological malignancies
}

\author{
Hana Geres*,1 \& Rouslan Kotchetkov² iD \\ ${ }^{1}$ Queen's University Belfast, Belfast, BT7 1NN, UK \\ ${ }^{2}$ Department of Hematology-Oncology, University of Toronto, Royal Victoria Regional Health Centre, Barrie, ON, L4M 6M2, Canada \\ *Author for correspondence: gereshana@gmail.com
}

\begin{abstract}
"Nurses are the first to encounter a patient during their time in hospital; whether it is in the emergency department, an outpatient setting, or as inpatients. As a result, nurses must be equipped with patient centered care models to manage a diversity of patients and conditions."
\end{abstract}

First draft submitted: 9 April 2020; Accepted for publication: 9 April 2020; Published online: 4 May 2020

Keywords: cancer side effects • hematology • nursing quality • oncology • quality of life

Managing patients battling hematological malignancies is a multifaceted profession and may occur within several settings. High quality nursing care demands an adequate understanding of the wide spectrum of diseases and their impact on a patient's ability to maintain homeostasis throughout the course of the disease. Impaired bone marrow function renders patients vulnerable to multiple complications. Nurses must be trained to care for patients with the appropriate additional precautions. They must be aware of common side effect profiles of varying treatment regimens and the signs and symptoms of hematological emergencies. Additionally, they facilitate important conversations between patients, their families and healthcare teams. This ensures that the psychosocial wellbeing of patients remains at the centre of all decisions and management plans.

\section{Art of nursing: advantages of specialized nursing care}

Malignant hematology is one of the most diverse fields in medicine. A diagnosis of a hematological malignancy (HM) can range from a chronic, indolent condition to a fast growing, often fatal disease. This vast spectrum of disease forces physicians and nurses to tailor their consultations to each of their patients unique medical history. Those with indolent diseases, such as chronic lymphocytic leukemia, are approached with a more relaxed management, often only requiring yearly active surveillance with blood count monitoring. However, those with faster progressing diseases, such as acute myeloid leukemia, are managed with a higher level of urgency. The tone of each consultation relies heavily on how healthcare practitioners approach the patient. Nurses are the first to encounter a patient during their time in hospital; whether it is in the emergency department, an outpatient setting, or as inpatients. As a result, nurses must be equipped with patient centered care models to manage a diversity of patients and conditions. Furthermore, it requires nurses to be familiar with the significance and likely sequelae of a patient's diagnosis, the common side effects of their treatment plans and the important factors for patient education. All of these domains will be discussed below.

\section{How to please sick bone marrow: importance of supportive care}

HM patients suffer from dysfunctional hematopoiesis due to impaired production or function of the bone marrow cells, secondary to the malignancy itself or to treatment regimens [1,2]. Progenitor cells often fail to fully mature or mature into dysmorphic variants and consequently cause one or multiple cytopenias [2]. Managing HM patients requires a compensatory increase in the services demanded from the healthcare system. Anemic patients will require additional transfusions, erythropoietic-stimulating agent administration, vigilant cardiac status monitoring and more, to ensure that their bodies are performing to the best of their abilities. Thrombocytopenic patients need to be monitored for signs of internal and mucocutaneous bleeding and often require frequent platelet transfusions. Nurses are often on the front lines, dealing with transfusion reactions. Leukopenic patients require additional aseptic protocols, infection control measures and often, granulocyte-colony stimulating factor injections [3]. These 
patients will also suffer from a heavy symptom burden. This is mainly attributed to their bone marrow's inability to maintain a normal level of homeostasis. The increased demand on our healthcare system equates to increased training and skills required from nursing staff to adequately contribute to caring for these patients. More specifically, nurses need to familiarise themselves with signs and symptoms that may aid in identifying the secondary effects of bone marrow suppression.

\section{Repel bugs from your patient! Care of the immunocompromised host}

Alongside bone marrow suppression, patients with hematological malignancies are also immunosuppressed secondary to neutropenia, lymphopenia and hypogammaglobulinemia. These conditions impair a patient's local defence systems by increasing vascular permeability, stasis and tissue damage, secondary to malignant invasion and anticancer therapies. As a result, nursing care is extended exponentially to accommodate for a patient's compromised immune system. Standard nursing tasks, such as central line care, drug administration and wound care, take longer to complete due to the added complexities of aseptic and isolation protocols on HM wards [4]. As these patients are more prone to infection, they require additional intravenous catheter, skin, mouth, wound and perineal care to maintain the same baseline health as nonimmunocompromised patients. These extenuating circumstances and their associated complications demand a specific and essential skillset from the nursing team.

\section{What to expect from the drugs? Knowledge of anticancer therapy side effects}

Patients with HM are often treated with strong, cytotoxic therapies to suppress or cure their conditions. These medications come with a substantial side effect profile, many of which need to be addressed urgently. As patients often spend the majority of their time with their nurses in outpatient chemotherapy suites or hospital inpatient wards, the burden of recognizing these adverse effects lies heavily on the nursing staff. Patients who are on an at-home oral therapies are also given a direct line to access a hematology nurse to offer them advice regarding side effects and address any of their concerns between clinic visits. General side effect profiles include nausea, diarrhea, fatigue, electrolyte disturbances and malnutrition secondary to decreased appetite and gastrointestinal dysfunction [5]. Additionally, monoclonal antibodies, such as rituximab and daratumumab, are associated with infusion reactions. Targeted therapy agents may have specific rare side effects, like dasatinib which is associated with pleural effusions. Doses of corticosteroids used to treat HM are usually much higher as compared with those in nonHM patients, thus the rates of hyperglycemia, skin atrophy, peptic ulcer disease, insomnia, mood changes and an increased risk of infections are subsequently significantly higher.

Nursing staff must also have a good understanding of hematological emergencies, most notably tumor lysis syndrome. HM patients are at a high risk of developing tumor lysis syndrome due to their rapid response to cytotoxic therapies, such as venetoclax in chronic lymphocytic leukemia patients. The rapid breakdown of malignant cells causes severe electrolyte imbalances, renal dysfunction and can be fatal. Patients feel ambiguously unwell thus early recognition and treatment is essential to improved patient outcomes [6]. The onus of educating patients falls largely on the nursing staff. Patients need to be given the autonomy to recognize adverse effects associated with their specific chemotherapy regimens. Nurses need to empower patients with the knowledge and resources to identify, manage and appropriately escalate medication reactions. HM patient care has evolved to improve patient outcomes by focusing treatment regimens in an outpatient setting [7]. This reduces the length of hospital stay and complications, however, it also shortens the patient's opportunity to raise any concerns. Nurses must build strong relationships with patients to promote open communication regarding quality of life $(\mathrm{QoL})$, response to treatment and side effect profiles [5]. Furthermore, patients must be encouraged to protect themselves from further complications. Nurses should discuss the daily lifestyle changes a patient should make in order to maintain their physical and mental health. These changes vary from frequent handwashing to dietary modifications, but must all be outlined in detail to the patient's understanding.

\section{Calm me down! How much psychosocial support is needed?}

Lastly, a patient's cancer journey is not limited to their in-hospital experience. Nurses can bridge the gap between a patient's psychosocial concerns and their healthcare team. From coping with the news of the diagnosis to adjusting to their new lifestyle, patients must feel welcome to raise their concerns and questions. Patient centered care focuses on involving patients and their caregivers in the decisions being made surrounding their care [8]. By facilitating open communication, nurses can address QoL issues, ease anxieties and enable good doctor-patient relationships. 
QoL is the most greatly affected domain in a patient's life following a cancer diagnosis, thus tailoring management plans to best suit a patient's lifestyle is essential [9].

Additionally, inpatients rely on nurses for a sense of consistency during their long hospital stays and outpatient visits. Stressful environments such as intensive care units, can be detrimental to a patient's mental health. This is especially true in cases where patients are separated from their usual carers and familiar support system [10]. Moreover, patients continue to rely on their nurses even after they've gone into remission. Anxieties surrounding potential relapse are common, especially if patients are on an 'active surveillance' protocol. Patients can rest assured that they can contact their primary nurses to discuss any questions or concerns during these uncertain times.

Both the psychological and physical wellbeing of a patient's health are heavily impacted by the nursing staff encountered throughout their medical journey. It is therefore, essential that all nursing staff are equipped and adequately trained to both identify signs of medical emergencies and tend to patient's emotional needs in their most vulnerable state. This combination of medical knowledge and communication skills will ensure that patients receive the highest quality of patient-centred medical care possible.

\section{Author contributions}

H Geres: supported research and wrote the main body of the manuscript; R Kotchetkov: designed and directed the project.

\section{Acknowledgments}

The authors thank our nursing team for providing ongoing care and support to our patients with hematological malignancies

\section{Financial \& competing interests disclosure}

The authors have no relevant affiliations or financial involvement with any organization or entity with a financial interest in or financial conflict with the subject matter or materials discussed in the manuscript. This includes employment, consultancies, honoraria, stock ownership or options, expert testimony, grants or patents received or pending or royalties.

No writing assistance was utilized in the production of this manuscript.

\section{Open access}

This work is licensed under the Attribution-NonCommercial-NoDerivatives 4.0 Unported License. To view a copy of this license, visit http://creativecommons.org/licenses/by-nc-nd/4.0/

\section{References}

1. Kemp K, Morse R, Wexler $\mathrm{S}$ et al. Chemotherapy-induced mesenchymal stem cell damage in patients with hematological malignancy. Ann. Hematol. 89, 701-713 (2010).

2. Hu D, Shilatifard A. Epigenetics of hematopoiesis and hematological malignancies. Genes \& Dev. 30, 2021-2041 (2016).

3. Hui D, Didwaniya N, Vidal M et al. Quality of end-of-life care in patients with hematologic malignancies: a retrospective cohort study. Cancer 120, 1572-1578 (2014).

4. Colombo A, Solberg B, Vanderhoeft E, Ramsay G, Schouten H. Measurement of nursing care time of specific interventions on a hematology-oncology unit related to diagnostic categories. Cancer Nurs. 28, 476-480 (2005).

5. Speksnijder H, Mank A, van Achterberg T. Nursing diagnoses (NANDA-I) in hematology-oncology: a delphi-study. Int. J. Nurs. Terminol. Classif. 22, 77-91 (2011).

6. Cairo M, Bishop M. Tumor lysis syndrome: new therapeutic strategies and classification. B. J. Haematol. 127, 3-11 (2004).

7. Wallis M, Tyson S. Improving the nursing management of patients in a hematology/oncology day unit. Cancer Nurs. 26, 75-83 (2003).

8. Tseng EK, Hicks LK. Value based care and patient-centered care: divergent or complementary? Curr. Hematol. Malig. Rep. 11, 303-310 (2016).

9. LeBlanc T, Abernethy A. Quality of life in higher resolution: the next generation of comparative effectiveness research in malignant hematology. Haematologica 98, 823-824 (2013).

10. McGrath P. Qualitative findings on the experience of end-of-life care for hematological malignancies. Am. J. Hosp. Palliat. Care 19, 103-111 (2002). 